\title{
Optical Microfiber Passive Components
}

\author{
Gilberto Brambilla \\ Optoelectronics Research Centre \\ University of Southampton \\ Southampton, United Kingdom \\ E-mail: gb2@orc.soton.ac.uk
}

\begin{abstract}
A review of passive components based on optical microfibers is presented.
\end{abstract}

Keywords- Optical fibre tapers; optical microfibers, fibre devices, optical fibre nanowires

\section{INTRODUCTION}

Optical microfibers (OMFs) are optical fibre tapers with a micrometric uniform waist region (fig. 1). They have also been labeled as optical microwires, photonic microwires and optical fibre microwires. OMFs are manufactured from optical fibres, thus they are connected by conical transition regions to the original optical fibres at their pigtails.

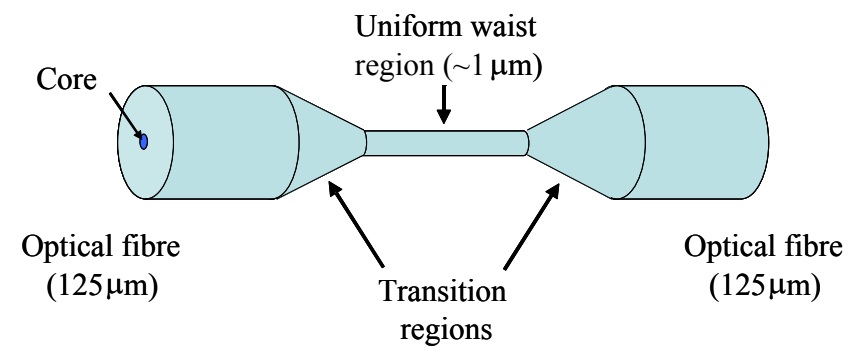

Figure 1. Schematic of an optical microfiber (OMF). The OMF (uniform wait region) is connected by two conical transition regions to its fibre pigtails which have the dimensions of a conventional optical fibre.

Interest in OMFs and related sensors has continuously grown, as the result of a publication in Nature in December 2003 showing that low-loss OMFs and nanofibres can be manufactured with a simple two-step process [1]. Even though the propagation loss of OMFs fabricated by this "selfmodulated taper-drawing" technique [1] was significantly higher than that recorded later in samples manufactured by the "flame-brushing" method [2-6], it was low enough to initiate research in a wide range of fields, delivering a large quantity of OMF devices.

Overall, OMFs have attracted so much attention because of the numerous extraordinary optical and mechanical properties that they offer:

1) strong confinement: when an OMF has a diameter comparable to half of the wavelength of the light transmitted in it, the propagating beam is confined by diffraction to its minimum waist diameter [8]

2) large evanescent fields: for small radii, a considerable fraction of the transmitted power can propagate in the evanescent field outside the OMF physical boundary [7]: OMF interferometers and resonating devices exploit this strong dependence of the evanescent field on the surrounding environment.

3) flexibility: because of their small stiffness, OMFs can stand micrometric bending radii $[9,10]$, providing the ultimate device compactness.

4) configurability: OMFs preserve the original optical fibre dimensions at their pigtails (fig. 1), allowing for low-loss interconnection to fiberized components and detectors.

5) robustness: OMFs have an extraordinary mechanical strength [11]. This allows for a relatively easy handling of a nanowire with macroscopic tools and equipment typical of the macroscopic world. OMF devices can also be embedded to provide sturdy devices which can be easily handled.

In the next sections a summary of OFN fabrication and properties will be presented, followed by an analysis of different typologies of devices, which will be categorized in three groups according to the property they exploit: confinement, evanescent field, resonators.

\section{DEVICE FABRICATION}

OMFs were fabricated mostly by one of the following methods:

1) The "self-modulated taper-drawing" [1]

2) The flame-brushing technique [2-6]

3) The modified flame-brushing technique $[9,12,13]$

4) Direct pull from bulk glass [14]

The "self-modulated taper-drawing" $[1,15]$ was the methodology firstly published in Nature in 2003. It is a twostep procedure: firstly, a taper with a diameter of few micrometers is drawn from an optical fibre using the conventional flame brushing technique; then, the manufactured taper is cut into two halves in the uniform waist region, and one of the microfibers is wrapped onto a hot sapphire rod and pulled to sub-micrometric diameters. The sapphire rod is heated by a flame positioned at a distance from the fibre, and it conveys the heat to the microfiber being pulled. Despite being complex and producing OMFs with a relatively high transmission loss, the self-modulated taper-drawing has provided OMFs with the smallest diameter: radii as small as 10 nm have been fabricated [15]. 
The "flame-brushing" technique [2-6] was originally developed for the fabrication of optical fibre tapers and couplers [16,17] and relies on a small flame moving under an optical fibre which is being stretched. The taper shape can be determined with a great accuracy by precisely controlling the flame movement and the fibre elongation. This technique has been used to manufacture the vast majority of OMFs reported in the literature.

The modified flame-brushing technique is derived, as the name suggests, from the flame brushing technique and it replaces the flame with a different heat source: a microheater $[12,13]$ or a sapphire capillary tube heated by a $\mathrm{CO}_{2}$ laser beam [9]. In the last few years it has emerged as the technique of choice for the manufacture of OMFs from compound glass optical fibres.

Finally, direct drawing from bulk [15] has been used to manufacture OMF from glasses with a relatively low softening temperature (like tellurites or ZBLAN). OMFs with diameters and losses as small as $50 \mathrm{~nm}$ and $0.1 \mathrm{~dB} / \mathrm{mm}$ have been reported. In this process a flame or a $\mathrm{CO}_{2}$ laser heat a thick sapphire fiber to a temperature at which the glass softens. The fiber is then immersed into the soft glass and removed from it with a portion of melt at its extremity. A second, smaller sapphire fiber is then brought into contact with the glass coated sapphire fiber and, as the glass temperature is reduced, it is withdrawn at a speed of $0.1-1 \mathrm{~m} / \mathrm{s}$ to draw wires from the melt.

\section{MODE PROPAGATION AND CONFINEMENT}

The weakly guiding approximation used in the derivation of the linearly polarised modes in conventional optical fibres cannot be used for OMFs because of the large refractive index difference at the interface between glass and air [18-19]. The exact solution of Maxwell's equation for hybrid modes $H E_{v m}$ and $E H_{v m}, T E_{0 m}$ and $T M_{0 m}$ give the following eigenvalue equations $[20,21]$ :

$$
\begin{aligned}
& {\left[\frac{J_{v}^{\prime}(U)}{U J_{v}(U)}+\frac{K_{v}^{\prime}(W)}{W K_{v}(W)}\right]\left[\frac{J_{v}^{\prime}(U)}{U J_{v}(U)}+\left(\frac{n_{\text {sur }}}{n_{\text {clad }}}\right)^{2} \frac{K_{v}^{\prime}(W)}{W K_{v}(W)}\right]=} \\
= & v^{2}\left(\frac{1}{U^{2}}+\frac{1}{W^{2}}\right)\left[\frac{1}{U^{2}}+\left(\frac{n_{\text {sur }}}{n_{\text {clad }}}\right)^{2} \frac{1}{W^{2}}\right] \\
& {\left[\frac{J_{1}(U)}{U J_{0}(U)}+\frac{K_{1}(W)}{W K_{0}(W)}\right]=0 } \\
& {\left[\frac{n_{1}^{2} J_{1}(U)}{U J_{0}(U)}+\frac{n_{2}^{2} K_{1}(W)}{W K_{0}(W)}\right]=0 }
\end{aligned}
$$

where $\mathrm{J}_{v}$ is the $v^{\text {th }}$ order Bessel function of the first kind, $\mathrm{K}_{v}$ is the $v^{\text {th }}$ order modified Bessel function of the second kind and $\mathrm{n}_{\text {clad }}$ and $\mathrm{n}_{\text {sur }}$ are the refractive indices of cladding and of the surrounding medium. The parameters $U$ and $W$ are defined as a function of the light propagation constant in vacuum $\left(k_{0}\right)$ and in the $\mathrm{OMF}(\beta)$ :

$$
\begin{aligned}
& U=r \sqrt{k_{0}^{2} n_{\text {clad }}{ }^{2}-\beta^{2}} \\
& W=r \sqrt{\beta^{2}-k_{0}^{2} n_{\text {surr }}^{2}}
\end{aligned}
$$

( $r$, is the OFM radius). $U$ and $W$ are related to the $\mathrm{V}$-number $(V)$, the OMF numerical aperture $N A$ and the wavelength $\lambda$ by:

$$
V=\sqrt{U^{2}+V^{2}}=\frac{2 \pi}{\lambda} r N A
$$

For $\mathrm{V}<2.405$ OMFs experience single mode guiding (as in common optical fibres), while for $\mathrm{V}>2.405$ the multimode operational range does not have degenerate modes $[19,20]$. As for optical fibres, $\mathrm{V}$ remains a useful parameter to evaluate the mode confinement properties: after experiencing a maximum confinement for $\mathrm{V} \sim 2$, the mode expands until it becomes orders of magnitude larger than $r$ for $V<0.6$ (fig. 2). The spot size $\omega$ of the beam propagating in an OFM is strongly dependent on $r$ [21]: since $\omega$ is minimum at $V \sim 2$ and $V$ is proportional to $N A$, different materials will experience a minimum $\omega$ at different $r$.

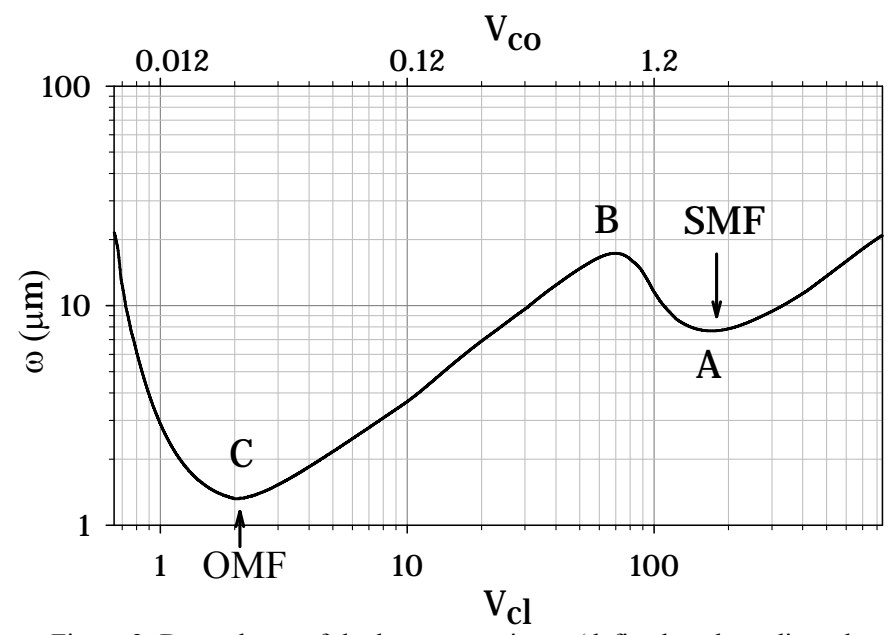

Figure 2. Dependence of the beam spot size $\omega$ (defined as the radius where the normalised intensity has dropped to $1 / \mathrm{e}^{2}[8,22]$ ) on the cladding $\mathrm{V}_{\mathrm{cl}}$ and core $\mathrm{V}_{\text {co }} \mathrm{V}$-numbers for a tapered telecom fibre. SMF and OMF represent the $\mathrm{V}$ numbers of a common telecom optical fibre and of an OMF with $r=500 \mathrm{~nm}$ in air at $\lambda=1.55 \mu \mathrm{m}$. A, B and $\mathrm{C}$ represent the points of maximum confinement in the core, of minimum confinement in the cladding and of maximum confinement in the cladding, respectively.

The combination of waveguide flexibility and tight mode confinement occurring at small $r$ implies that OMFs can be subjected to small bending radii $\rho$ without any significant loss: microcoils with $\rho<100 \mu \mathrm{m}$ reported in the literature were virtually lossless [8,21]. If $r$ becomes very small (at the far left of $\mathrm{C}$ in fig. 2) bending losses start to be an issue; in fact they have an exponential dependence on the ratio $\rho / r$ and on $V^{l}$ [9,23], which increase considerably for decreasing $r$. In high refractive index materials, the tighter mode confinement implies that even smaller $\rho$ can be achieved for the same $r$.

\section{DEVICES BASED ON CONFINEMENT}

Strong modal confinement enhances the waveguide optical nonlinearity $\gamma=\frac{2 \pi}{\lambda} \cdot \frac{n_{2}}{A_{\text {eff }}}\left(n_{2}\right.$ and $\mathrm{A}_{\text {eff }}$ are the material nonlinear 
refractive index and the beam effective area, respectively) and allows for the prompt observation of nonlinear effects like supercontinuum generation [24-34], third-harmonic generation $[21,35]$, slow and fast light [36] and bistability [37,38]. While standard telecom single-mode fibres (SMFs) have $\gamma \sim 10^{-3} \mathrm{~W}^{-1} \mathrm{~m}^{-}$ 1, OMFs manufactured from them have $\gamma \sim 70 \cdot 10^{-3} \mathrm{~W}^{-1} \mathrm{~m}^{-1}$, because of the one-order-of-magnitude-reduction in $\omega$. When highly nonlinear materials are used to manufacture OMFs, $\gamma$ can reach $\gamma \sim 10^{2} \mathrm{~W}^{-1} \mathrm{~m}^{-1}$ [34]. Highly nonlinear glasses usually are transparent far into the infrared region, thus they can be exploited for the generation of supercontinuum in spectral regions where silica is not transparent [29].

\section{A. Supercontinuum Generation}

Supercontinuum generation encompasses a variety of nonlinear effects, including self- and cross-phase modulation, four-wave mixing, Raman scattering, and soliton generation. In general, materials with higher $\gamma$ require lower laser peak powers to observe spectral broadening. Although compound glass fibres and silica microstructured fibres have been used to generate supercontinuum, they present high insertion losses because of the small core/high refractive index. In contrast, OMFs provide lossless connection to fiberized sources and components and the possibility to tailor the waveguide dispersion at any wavelength by changing the waveguide diameter. Supercontinua have been generated over a range of wavelengths several hundred $\mathrm{nm}$ wide in silica [28,29], bismuth-silicate [33] and chalcogenide [34] OMFs. Fig. 3 shows supercontinuum generated by fs pulses in a bismuth silicate OMF. The generated supercontinuum is very smooth and it extends for over $1000 \mathrm{~nm}$ with a $3 \mathrm{~dB}$ spectral width of $\sim 700 \mathrm{~nm}$.

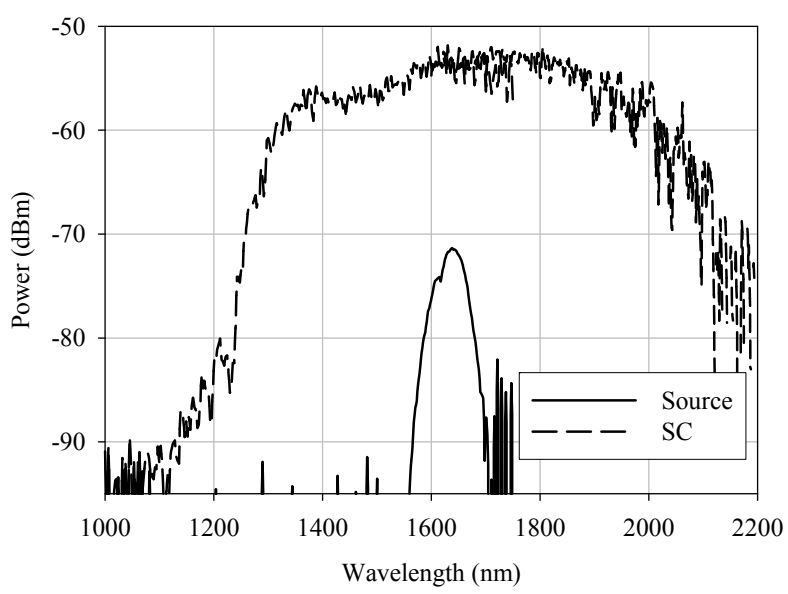

Figure 3. Supercontinuum generated by $\sim 1 \mathrm{~nJ}, 150 \mathrm{fs}$ pulses at $\lambda \sim 1630 \mathrm{~nm}$ in a bismuth silicate OMF. The OMF radius was $r \sim 1.6 \mu \mathrm{m}$, corresponding to a zero-dispersion wavelength $\mathrm{ZDW} \sim 1.63 \mu \mathrm{m}$.

\section{B. High harmonics generation}

Generation of high harmonics requires both phase matching and high overlap between modes at different wavelengths. Although phase matching is difficult to satisfy in conventional fibres because of the material dispersion, in OMF a considerable fraction of the mode is propagating in the evanescent field and this can provide both high overlap and phase matching between the fundamental frequency and its third harmonic: for optimised diameters, the overlap can reach $70 \%$ in phase matching conditions [21]. Indeed, phase matching between the two frequencies can be seen as a relation between the effective indices of the fundamental and third harmonic ( $n_{\text {eff }}^{\omega}$ and $n_{\text {eff }}^{3 \omega}$ ). The fundamental frequency can be phase matched to its higher-harmonics because high order modes extend more than the fundamental mode into the surrounding medium, which has a lower refractive index than glass and thus compensates for the contribution of the glass refractive index increase at shorter wavelengths. Fig. 4 presents the dependence of $n_{\text {eff }}^{\omega}$ and $n_{\text {eff }}^{3 \omega}$ from $r$ and shows that phase matching occur at $r=253 \mathrm{~nm}$ for THG and at $262 \mathrm{~nm}$ for SHG. Overlaps greater than $50 \%$ have been predicted [21].

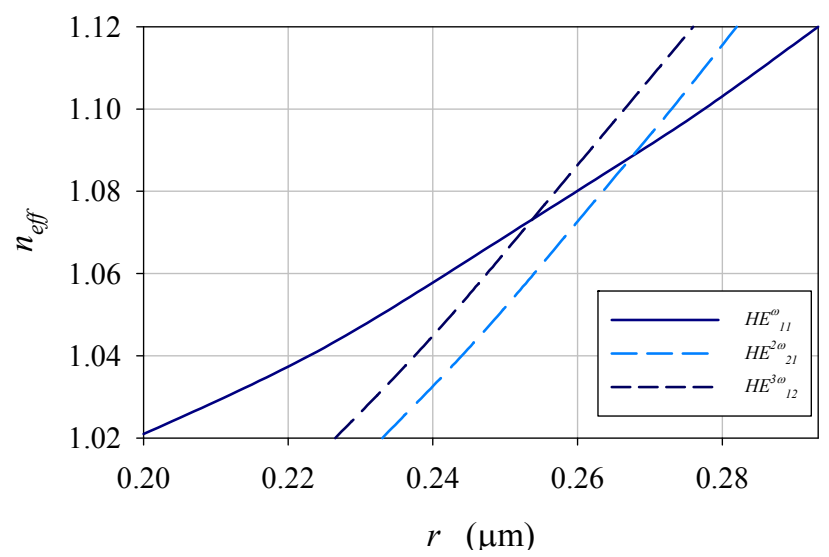

Figure 4 Relation between the effective index $n_{\text {eff }}$ of the modes propagating in the OMF and the OMF radius $r$ at $\lambda=1.06 \mu m$. $H E^{\omega}{ }_{11}, H E^{2 \omega}{ }_{2 l}$ and $H E^{3 \omega}{ }_{12}$ represent the $\mathrm{HE}_{11}$ mode at the fundamental frequency, the $\mathrm{HE}_{21}$ mode at the second-harmonic and the $\mathrm{EH}_{12}$ mode at the third-harmonic, respectively.

\section{Devices based on confinement in cleaved OMFs}

Devices based on confinement in cleaved OMFs include optical traps, sub-wavelength confinement and sensing. Rapid divergence of beams propagating in cleaved OMFs and the related large gradients in the intensity profile have been exploited to trap particles [39]. Although end-face lensing or shaping is possible, its divergence in the near field is only mildly affected by these structures. Generation of subwavelength spot sizes has been achieved converting light into surface plasmon polaritons (SPP) and then back into light [40]. Minimally invasive point sensors have been manufactured exploiting functionalised OMF with extremely small cross section [41-43]. Metal coated tips with radii $r \sim 50-500 \mathrm{~nm}$ had the end face uncoated and derivatized to immobilize biorecognition molecules. $\mathrm{pH}$, biochemicals (like benzopyrene tetrol, and benzo[ $\alpha]$ pyrene cytochrome c, caspase-9) and ions (like potassium, calcium or nitric acid) were measured in-vivo in real time in a single cell.

\section{EVANESCENT FIELD}

For $\mathrm{V}<<2$, the mode is weakly bound to the OMF and most of the power propagates in the evanescent field. The fraction of the propagating power inside the OMF can be obtained from 
the Poynting component in the propagation direction $S_{z}$ $[19,20]$. Since mode confinement is dependent both on the OMF refractive index and the OMF radius, it is convenient to consider the dependence on $V$. Simulations showed that for $V \sim 1 \quad \eta \sim 0.06$, meaning that $\sim 94 \%$ of the power is propagating outside the OMF physical boundary, in the evanescent field. The extension of the evanescent field depends on the ratio $\lambda / r$ between the wavelength of the radiation propagating in the OMF and the OMF radius [8]. For $\lambda / r \sim 10$, the evanescent field intensity decreases to $1 / 10$ of its maximum value over a distance two orders of magnitude larger than $r$. The magnitude of the evanescent field increases for increasing $\lambda / r$ and $n_{\text {surr }}$ (refractive indices of the medium surrounding the OMF). This effect can be exploited for the realization of sensors and high-Q resonators.

\section{DEVICES BASED ON EVANESCENT FIELD}

Most of the devices based on OMF fall in this category. They can be broadly classified in linear or resonant devices.

\section{A. Linear devices}

A large fraction of the mode in the OMF can propagate outside the OMF and overlap with the surrounding environment; thus any change in the environment properties results in a change at output. For OMF sensors the surrounding environment is simply a fluid, for interferometers and interferometric devices, the surrounding environment includes another OMF.

Linear sensors include 1) refractometric sensors, where any change of the fluid properties is recorded as a function of its related refractive index change, and 2) absorption sensors, where changes in the fluid properties are related to intensity changes, thus OMF transmissivity changes. In both cases the OMF surface can be functionalised to selectively bind specific compounds [41]. When linear sensors are inserted in one arm of Mach-Zehnder interferometers, their sensitivity is considerably increased and by monitoring the phase change at the interferometer output extremely high sensitivities have been predicted [44]. Indeed, Mach-Zehnder interferometers with extinction ratio as large as $10 \mathrm{~dB}$ have been shown both in silica and tellurite fibres [45].

An OMF coated with palladium was used for the detection of hydrogen in the range $0.05 \%-5 \%$, sufficient to detect hydrogen in gas mixtures at the lower explosion limit. OMFs coated with gelatine were used to detect humidity in the range 9-94\% RH [46] within few tens of ms. Polymer OMF have also been used for humidity, $\mathrm{NO}_{2}$ and $\mathrm{NH}_{3}$ sensing [47]. Polymers have been used as sensing media because they can absorb gas and their optical properties change when impregnated by gases. Bare silica OMFs were used to detect sub-monolayers of chemicals absorbed on their surface [48]. Molecules and their agglomeration dynamics were recorded on a second to minute scale. It has been shown that very small number of atoms (average atom number of 0.07) can be detected under strong resonant laser irradiation. Finally, OMFs were also deployed in microfluidic channels for the refractive index measurement [49]: an OMF was immersed in a transparent curable polymer in proximity of a fluidic channel: the minimum measurable refractive index change was estimated to be $5 \cdot 10^{-4}$.

Since the extent of the evanescent field is large, the field in proximity of the surface can be used to manipulate particles: OMF have been used to propel polystyrene microspheres with $3 \mu \mathrm{m}$ - [50] and $10 \mu \mathrm{m}-$ [51] diameter and microsphere clusters with diameters larger than $20 \mu \mathrm{m}$ [50] with speeds as high as $10 \mu \mathrm{m} / \mathrm{s}$.

OMF have also been proposed as atom guides [52]. Cs atoms at a temperature $\mathrm{T}<0.29 \mathrm{mK}$ were trapped and propelled along an OMF with $\mathrm{r}=200 \mathrm{~nm}$ a laser delivering $27 \mathrm{~mW}$ at $\lambda=1.3 \mu \mathrm{m}$. Because of the closeness of the atoms to the $\mathrm{OMF}$ surface, the OMF guided mode can collect the single-atom fluorescence.

\section{B. Resonators and resonating devices}

By coiling an OMF onto itself, modes in the adjacent sections can overlap and couple, creating high-Q resonators with extremely compact geometries. Three types of OMF resonators have been proposed and realized in the literature: knot, loop and microcoil.

Knot resonator: knot resonators (KRs) have been the first OMF resonators to be experimentally demonstrated [1]. Fig. 5a shows the schematic of a knot resonator. KRs are easy to manufacture and display an enhanced stability because of the friction between different adjacent sections of the OMF. Still, OMFs often break during fabrication, and exploit evanescent field for input/output coupling.

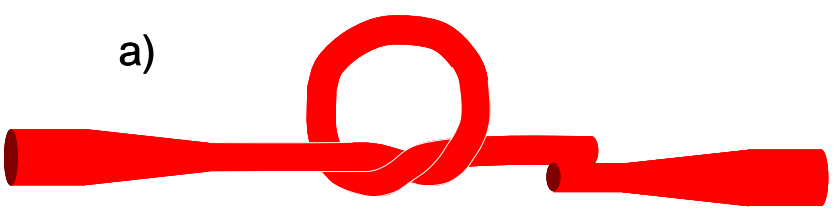

b)

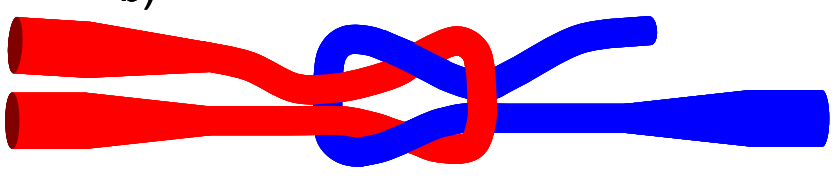

c)

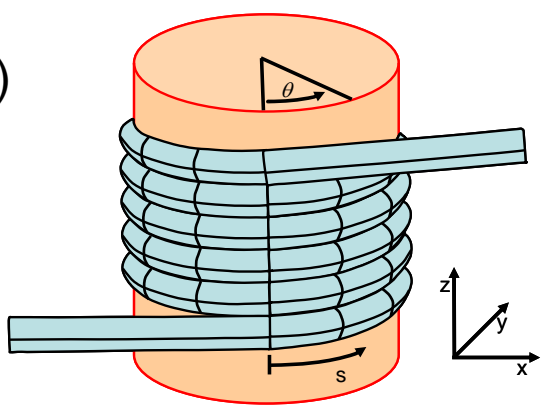

Figure 5 Schematic of (a) a knot resonator, (b) a reef knot resonator, and (c) a microcoil resonator. 
Q factors as high as 57000 with finesse of 22 have been demonstrated in KR with knot diameters of the order of few hundred $\mu \mathrm{m}$. Embedding in a range of polymers showed that extinction ratio and Q-factor are preserved during embedding. Lasing has been observed for KR made from active media: a 2mm-diameter KR, manufactured from Er:Yb-doped phosphate glass OMF with $\mathrm{r} \sim 1.9 \mu \mathrm{m}$, lased at $\lambda=1.5 \mu \mathrm{m}$ with $5 \mathrm{~mW}$ threshold and $\sim 8 \mu \mathrm{W}$ output power [53]: at critical coupling enhancements as large as 200 can occur, thus $\mu \mathrm{W}$ threshold pump powers and large increases in quantum efficiency can be expected. A fraction of the power is propagating outside the OMF, thus external lasing media (like rhodamine $6 \mathrm{G})$ or semiconductor nanowires $(\mathrm{ZnO})$ can be used. Add/drop filters have been fabricated by using multiple OMF to inject/extract light from a $\sim 308 \mu \mathrm{m} \mathrm{KR}$ with $\mathrm{Q} \sim 13,000$ and a free spectral range (FSR) of $1.8 \mathrm{~nm}$ [54]. At high powers KR can exhibit hysteresis cycles, depending on the cavity detuning and the input scanning frequency [37]. These were attributed to nonlinear phase shifts of thermal origin. Simulations showed that for silica the threshold for bistability is predicted to be in excess of $10 \mathrm{~W}$.

A second type of KR has been recently demonstrated [55]: the reef knot resonator (RKR) schematic is shown in fig. 5b. RKR has numerous advantages, including multiple input/output ports and the possibility to make hybrid resonators: a RKR was manufactured from silica and chalcogenide OMFs and exhibited $Q \sim 10^{4}$.

Loop resonator: loop resonators (LRs) are the simplest OMF resonators [56] and are easy to manufacture from OMFs by coiling them into a self-touching loops [57]. The first demonstration of a $2 \mathrm{~mm}$ diameter LR was carried out with an OMF having $\mathrm{r} \sim 4.2 \mu \mathrm{m}[58]$ : since the OMF size was too large to ensure sufficient self coupling, the LR was embedded into a silicone-rubber. In free space, an OMF with $\mathrm{r} \sim 500 \mathrm{~nm}$ was used to demonstrate a LR with a Q-factor of 95000 [57]. In air the LR shape is maintained because of electrostatic and van der Waals interactions between the different parts of the coil, thus the LR temporal stability is strongly related to the environmental conditions.

LR properties can be derived analytically [57] by solving the coupled wave equations for the OMF. If the LR is assumed uniform, the loop length is $L=2 \pi R_{0}$ (where $R_{0}$ is the loop radius) the propagation constant $\beta$ is independent from the position, and the resonating condition (minima of $|T|$ ) satisfies:

$$
\beta R_{0}=n_{\text {eff }} \frac{L}{\lambda}=N
$$

( $N$ is an integer). Eq. 7 allows to predict the optimized LR design. In practice, the LR optical properties can easily be recorded in real time by launching light from a broadband source into the OMF pigtail and analyzing the transmitted light with an optical spectrum analyzer: OMF can be manipulated until the desired spectrum is obtained.

LRs have been proposed as refractometric sensing devices for biological applications [59]: refractive indices smaller than $10^{-5}$ can be evaluated for OMF with $\mathrm{r} \sim 300 \mathrm{~nm}$ at $\lambda=1.55 \mu \mathrm{m}$. Embedded LR sensors have be considered to provide long term stability together with enhanced performances [60]: sensitivities exceeding $10^{3} \mathrm{RIU} / \mathrm{nm}$ have been predicted at $\lambda=1.55 \mu \mathrm{m}$ for $\mathrm{r} \sim 300 \mathrm{~nm}$. Copper-rod- supported LRs have also been proposed as sensors [61] because of their robustness and the flexibility of obtaining critical coupling within a broad spectral range: the estimated minimum measurable refractive index was $\sim 10^{-5}$. The use of a copper rod as support for the LR provides a great advantage: the resonance wavelength can simply be tuned by injecting an electric current in the copper wire [62]; an approximately-linear wavelength shift was measured with respect to the electric current with a slope of $\sim 26.5 \mathrm{pm} / \mathrm{A}$. A LR with an extinction ratio of $30 \mathrm{~dB}$ and Q 4000 was demonstrated by tuning the coupling coefficient.

Microcoil resonator: The microcoil resonator (MR) is a $3 \mathrm{D}$ OMF resonator (fig. 5c). MR is self-coupling along the whole coil. MR were first proposed in 2004 [56] and realized experimentally in 2007 [63]. The MR transmission spectrum is strongly dependent on its geometry: a small change in the relative position of the $\mathrm{OMF}$ in the coils can radically change the resonance shape and shift its frequency. When coupling between non-adjacent turns is ignored, the analytical transmission properties can be predicted using the coupledwave equations [56]. Although MRs with large numbers of turns have the best theoretical performances, simulations with practical losses showed that high-Q resonators can be obtained even for resonators with only three or four turns.

Because of their intrinsically high stability, MRs are well suited for sensing applications. Refractometric sensors have been proposed [64] and demonstrated [65] from embedded MR. They are compact and robust devices with an intrinsic fluidic channel to deliver samples to the sensor. The embedded OMF has a considerable fraction of its mode propagating in the fluidic channel, thus any change in the analyte properties is reflected in a change of the properties of the mode propagating in the OMF. At the MR sensor output, a shift in the resonant wavelength is associated to a change in the analyte refractive index. The wavelength shift is particularly affected by the wavelength, by the OMF radius and by the coating thickness $d$ between the OMF and the fluidic channel. Sensitivities as high as $S=10^{3} \mathrm{~nm} / \mathrm{RIU}$ (refractive index unit) have been predicted in refractometric MR sensors [64]. An alternative refractometric resonating sensor has been proposed by using a microfluidic capillary to excite whispering gallery modes and an OMF to launch and collect the signal [66]. The sensor, named liquidcore optical ring-resonator sensor, was embedded into a low refractive index polymer to increases the overlap between the whispering gallery mode excited in the capillary wall and the analyte flowing in the capillary. Since the sensor sensitivity is strongly dependent on the overlap between the mode and the analyte, it is also related to the analyte refractive index $n_{a}$ : for $n_{a} \sim 1.3$ (close to the water refractive index) $S \sim 40 \mathrm{~nm} / \mathrm{RIU}$, while for $n_{a}>1.5, S \sim 800 \mathrm{~nm} / \mathrm{RIU}$ can be expected.

MRSs have also been proposed as effective means to detect rotation [67] from the phase difference between the co- and counter-propagating beams in the resonator. In MRs, slow-light and conventional mode propagation augment the Sagnac phase shift by orders of magnitudes in comparison to conventional optical gyroscopes. It is possible to enhance sensitivity to rotation if the MR gyroscope operates at wavelengths close to 
the spectral resonances, where the phase changes rapidly. For increasing coupling within the MR coils, the MR gyroscope increases its sensitivity and enhancements in excess of three orders of magnitude have been predicted for small losses.

\section{CONCLUSION}

In conclusion, the large fraction of power propagating in the evanescent field, jointly to the extreme flexibility and configurability allows the prompt use of microfibers for a variety of optical passive applications ranging from high-Q resonators to interferometers, supercontinuum generators and sensors.

\section{REFERENCES}

[1] L.M. Tong, R.R. Gattass, J.B. Ashcom, S.L. He, J.Y. Lou, M.Y. Shen, I. Maxwell, E. Mazur, Subwavelength-diameter silica wires for low-loss optical wave guiding, Nature 426 (2003) 816-9.

[2] G. Brambilla, V. Finazzi, D.J. Richardson, Ultra-low-loss optical fiber nanotapers, Opt. Express 12 (2004) 2258-63.

[3] S.G. Leon-Saval, T.A. Birks, W.J. Wadsworth, P.St.J. Russell, M.W. Mason, Supercontinuum generation in submicron fibre waveguides, Opt. Express 12 (2004) 286469.

[4] A.M. Clohessy, N. Healy, D.F. Murphy, C.D. Hussey, Short low-loss nanowire tapers on singlemode fibres, Electron. Lett. 41 (2005) 27-29.

[5] G. Brambilla, F. Xu, X. Feng, Fabrication of optical fibre nanowires and their optical and mechanical characterisation, Electron. Lett. 42 (2006) 517-8.

[6] G. Brambilla, Optical fibre nanowires and microwires: a review, J. Opt. (2010).

[7] L. Tong, J. Lou, E. Mazur, Single-mode guiding properties of subwavelength-diameter silica and silicon wire waveguides, Opt. Express 12 (2004) 1025-35.

[8] G. Brambilla, F. Xu, P. Horak, Y. Jung, F. Koizumi, N.P. Sessions, E. Koukharenko, X. Feng, G.S. Murugan, J.S. Wilkinson, D.J. Richardson, Optical fiber nanowires and microwires: fabrication and applications, Adv. Opt. Photon. 1 (2009) 107-61.

[9] M. Sumetsky, Y. Dulashko, and A. Hale, Fabrication and study of bent and coiled free silica nanowires: Self-coupling microloop optical interferometer, Opt. Express 12 (2004) 3521-31.

[10] L. Tong, J. Lou, R. R. Gattass, S. He, X. Chen, L. Liu, and E. Mazur, Assembly of silica nanowires on silica aerogels for microphotonic devices, Nano Lett. 5 (2005) 259-2.

[11] G. Brambilla, D.N. Payne, The Ultimate Strength of Glass Silica Nanowires, Nano Lett. 9 (2009) 831-5.

[12] G. Brambilla, F. Koizumi, X. Feng, and D.J. Richardson, Compound-glass optical nanowires, Electron. Lett. 41 (2005) 400-2.

[13] E.C. Mägi, L.B. Fu, H.C. Nguyen, M.R.E. Lamont, D.I. Yeom, and B.J. Eggleton, Enhanced Kerr nonlinearity in subwavelength diameter $\mathrm{As}_{2} \mathrm{Se}_{3}$ chalcogenide fiber tapers, Opt. Express 15(2007) 10324-9.

[14] L. Tong, L. Hu, J. Zhang, J. Qiu, Q. Yang, J. Lou, Y. Shen, J. $\mathrm{He}$, and $\mathrm{Z}$. Ye, Photonic nanowires directly drawn from bulk glasses, Opt. Express 14 (2006) 82-87

[15] L.M. Tong, J.Y. Lou, Z.Z. Ye, T.S. Geoff, and E. Mazur, Self-modulated taper drawing of silica nanowires, Nanotechnol. 16 (2005) 1445-8.

[16] F. Bilodeau, K.O. Hill, S. Faucher, and D.C. Johnson, Lowloss highly overcoupled fused couplers - Fabrication and sensitivity to external pressure, J. Lightwave Technol. 6 (1988) 1476-82.

[17] T.A. Birks, and Y.W. Li, The shape of fiber tapers, J. Lightwave Technol. 10 (1992) 432-8.

[18] K. Okamoto, Fundamentals of Optical Waveguides 2000 Academic Press.

[19] L. Tong, J.Y. Lou, and E. Mazur, Single-mode guiding properties of subwavelength-diameter silica and silicon wire waveguides, Opt. Express 12 (2004), 1025-35.

[20] F. Biancalana, and D.V. Skryabin, Vector modulational instabilities in ultra-small core optical fibres J. Opt. A: Pure Appl. Opt. 6 (2004) 301-306

[21] V. Grubsky, and A. Savchenko, Glass micro-fibers for efficient third harmonic generation, Opt. Express 13 (2005) 6798-6806.

[22] J.D. Love, Spot size, adiabaticity and diffraction in tapered fibers, Electron. Lett. 23 (1987) 993-4.

[23] H. Yu, S. Wang, J. Fu, M. Qiu, Y. Li, F. Gu, and L. Tong, Modeling bending losses of optical nanofibers or nanowires, Appl. Opt. 48 (2009) 4365-4369.

[24] H. Ebendorff-Heidepriem, S. C. Warren-Smith, and T. M. Monro, Suspended nanowires: fabrication, design and characterization of fibers with nanoscale cores, Opt. Express 17 (2009) 2646-57.

[25] J.Y.Y. Leong, P. Petropoulos, J.H.V. Price, H. EbendorffHeidepriem, S. Asimakis, R.C. Moore, K.E. Frampton, V. Finazzi, X. Feng, T.M. Monro, and D.J. Richardson, HighNonlinearity Dispersion-Shifted Lead-Silicate Holey Fibers for Efficient 1- $\mu \mathrm{m}$ Pumped Supercontinuum Generation, J. Lightwave Technol. 24 (2006) 183-90.

[26] Y. Xu, X. Chen, and Y. Zhu, Modeling of micro-diameterscale liquid core optical fiber filled with various liquids, Opt. Express 16 (2008) 9205-12.

[27] J.H.V. Price, T.M. Monro, H. Ebendorff-Heidepriem, F. Poletti, P. Horak, V. Finazzi, J.Y.Y. Leong, P. Petropoulos, J.C. Flanagan, G. Brambilla, X. Feng, and D.J. Richardson, Mid-IR Supercontinuum Generation From Nonsilica Microstructured Optical Fibers, IEEE J. Sel. Top. Quantum Electron. 13 (2007) 738-49.

[28] T.A. Birks, W.J. Wadsworth, and P.St.J. Russell, Supercontinuum generation in tapered fibers, Opt. Lett. 25, (2000) 1415-17.

[29] C.M.B. Cordeiro, W.J. Wadsworth, T.A. Birks, and P.St.J. Russell, Engineering the dispersion of tapered fibers for supercontinuum generation with a $1064 \mathrm{~nm}$ pump laser, Opt. Lett. 30 (2005) 1980-2.

[30] R. Zhang, J. Teipel, X. Zhang, D. Nau, and H. Giessen, Group velocity dispersion of tapered fibers immersed in different liquids, Opt. Express 12, (2004) 1700-7.

[31] J. Lou, L. Tong, and Z. Ye, Dispersion shifts in optical nanowires with thin dielectric coatings, Opt. Express 14, (2006) 6993-98.

[32] J.J. Hu, P.P. Shum, G. Ren, X. Yu, G. Wang, and C. Lu, Investigation on the interplay between dispersion and nonlinearity in subwavelength-diameter silica fibers, Microwave Opt. Technol. Lett. 50 (2008) 2086-90.

[33] G. Brambilla, F. Koizumi, V. Finazzi, and D.J. Richardson, Supercontinuum generation in tapered bismuth silicate fibres, Electron. Lett. 41 (2005) 795-797.

[34] D. Yeom, E.C. Mägi, M.R.E. Lamont, M.A.F. Roelens, L. $\mathrm{Fu}$, and B.J. Eggleton, Low-threshold supercontinuum generation in highly nonlinear chalcogenide nanowires, Opt. Lett. 33 (2008) 660-2.

[35] V. Grubsky, and J. Feinberg, Phase-matched third-harmonic UV generation using low-order modes in a glass micro-fiber, Opt. Commun. 274 (2007) 447-50. 
[36] N.G. Broderick, Optical Snakes and Ladders: Dispersion and nonlinearity in microcoil resonators, Opt. Express 16 (2008) 16247-54.

[37] G. Vienne, Y. Li, L. Tong, and P. Grelu, Observation of a nonlinear microfiber resonator, Opt. Lett. 33 (2008) 1500-2

[38] G. Vienne, P. Grelu, X. Pan, Y. Li, and L. Tong, heoretical study of microfiber resonators exploiting a phase shift, J. Opt. A: Pure Appl. Opt. (2008) 10025303.

[39] G. Brambilla, and F. Xu, Adiabatic submicrometric tapers for optical tweezers, Electron. Lett. 43(2006) 204-5.

[40] F. Renna, D. Cox, and G. Brambilla, Efficient subwavelength light confinement using surface plasmon polaritons in tapered fibers, Opt. Express 17(2009) 7658-63.

[41] T. Vo-Dinh, J.P. Alarie, B.M. Cullum, and G.D. Griffin, Antibody-based nanoprobe for measurement of a fluorescent analyte in a single cell, Nat. Biotechnol. 18 (2000) 764-7.

[42] B. Cullum, G.D. Griffin, G.H. Miller, and T. Vo-Dinh, Intracellular Measurements in Mammary Carcinoma Cells Using Fiber-optic Nanosensors, Anal. Biochem. 277 (2000), 25-32.

[43] T. Vo-Dinh, and B. Cullum, Biosensors and biochips: advances in biological and medical diagnostics, Fresenius J. Anal. Chem. 366 (2000) 540-51.

[44] J.Y. Lou, L.M. Tong, and Z.Z. Ye, Modeling of silica nanowires for optical sensing, Opt. Express 13 (2005) 213540

[45] H.Y. Li, L.M. Tong, Mach-Zehnder interferometers assembled with optical microfibers or nanofibers Opt. Lett. 33 (2008) 303-5.

[46] L. Zhang, F. Gu, J. Lou, X. Yin, and L. Tong, Fast detection of humidity with a subwavelength-diameter fiber taper coated with gelatin film, Opt. Express 16 (2008) 13349-53.

[47] F.X. Gu, L. Zhang, X.F. Yin, and L.M. Tong, Polymer single-nanowire optical sensors, Nano Lett. 8(2008) 275761.

[48] P. Polynkin, A. Polynkin, N. Peyghambarian, and M Mansuripur, Evanescent field-based optical fiber sensing device for measuring the refractive index of liquids in microfluidic channels, Opt. Lett. 30 (2005) 1273-5.

[49] F. Warken, E. Vetsch, D. Meschede, M. Sokolowski, and A. Rauschenbeutel, Ultra-sensitive surface absorption spectroscopy using sub-wavelength diameter optical fibers, Opt. Express 15(2007) 11952-8

[50] G. Brambilla, G.S. Murugan, J.S. Wilkinson, and D.J. Richardson, Optical manipulation of microspheres along a subwavelength optical wire, Opt. Lett. 32(2007) 3041-3.

[51] G.S. Murugan, G. Brambilla, J.S. Wilkinson, and D.J. Richardson, Optical propulsion of individual and clustered microspheres along sub-micron optical wires, Jap. J. Appl. Phys. 47(2008) 6716-8.

[52] V.I. Balykin, K. Hakuta, F. Le Kien, J.Q. Liang, and M. Morinaga, Atom trapping and guiding with a subwavelengthdiameter optical fiber, Phys. Rev. A 70(2004) 011401.

[53] X.S. Jiang, Q. Yang, G. Vienne, Y.H. Li, L.M. Tong, J.J. Zhang, and L.L. Hu, Demonstration of microfiber knot laser, Appl. Phys. Lett. 89(2006) 143513.

[54] X.S.Jiang, Y. Chen, G. Vienne, L.M. Tong, Theoretical study of microfiber resonator devices exploiting a phase shift, Opt. Lett. 32(2007) 1710-2.

[55] G. Vienne, A. Coillet, P. Grelu, M. El Amraoui, J.C. Jules, F. Smektala, and L.M. Tong, Demonstration of a reef knot microfiber resonator, Opt. Express 17(2009) 6224-9.

[56] M. Sumetsky, Optical fiber microcoil resonators, Opt. Express 12(2004) 2303-16

[57] M. Sumetsky, Y. Dulashko, J.M. Fini, and A. Hale, Optical microfiber loop resonator, Appl. Phys. Lett. 86(2005) 161108.

[58] C. Caspar, and E.J. Bachus, Fibre-optic micro-ring-resonator with 2 mm diameter, Electron. Lett. 25(1989) 1506-8.

[59] L. Shi, L. Xu, W. Tan, and X. Chen, Simulation of optical microfiber loop resonators for ambient refractive index sensing, Sensors 7(2007) 689-96.

[60] F. Xu, V. Pruneri, V. Finazzi, and G. Brambilla, An embedded optical nanowire loop resonator refractometric sensor, Opt. Express 16 (2008) 1062-7.

[61] X. Guo, and L.M. Tong, Supported microfiber loops for optical sensing, Opt. Express 16(2008) 14429-34.

[62] X. Guo, Y. Li, X. Jiang, and L.M. Tong, Demonstration of critical coupling in microfiber loops wrapped around a copper rod, Appl. Phys. Lett. 91(2007) 073512.

[63] F. Xu, and G. Brambilla, Manufacture of 3-D microfiber coil resonators, Photon. Technol. Lett. 19(2007) 1481-3.

[64] F. Xu, P. Horak, and G. Brambilla, Optical microfiber coil resonator refractometric sensor, Opt. Express 15(2007) 788893.

[65] F. Xu, and G. Brambilla, Demonstration of a refractometric sensor based on optical microfiber coil resonator, Appl. Phys. Lett. 92 (2008) 101126.

[66] M. Sumetsky, R.S. Windeler, Y. Dulashko, and X. Fan, Optical liquid ring resonator sensor, Opt. Express 15(2007) 14376-81.

[67] J. Scheuer, Fiber microcoil optical gyroscope, Opt. Lett. 34(2009) 1630-2. 